\title{
Development of a redundant anthropomorphic hydraulically actuated manipulator with a roll-pitch-yaw spherical wrist
}

\author{
(C) The Author(s) 2021. This article is published with open access at link.springer.com and journal.hep.com.cn
}

\begin{abstract}
The demand for redundant hydraulic manipulators that can implement complex heavy-duty tasks in unstructured areas is increasing; however, current manipulator layouts that remarkably differ from human arms make intuitive kinematic operation challenging to achieve. This study proposes a seven-degree-of-freedom (7-DOF) redundant anthropomorphic hydraulically actuated manipulator with a novel roll-pitch-yaw spherical wrist. A hybrid series-parallel mechanism is presented to achieve the spherical wrist design, which consists of two parallel linear hydraulic cylinders to drive the yaw/pitch 2DOF wrist plate connected serially to the roll structure. Designed as a 1RPRRR-1SPU mechanism ("R", "P", "S", and "U" denote revolute, prismatic, spherical, and universal joints, respectively; the underlined letter indicates the active joint), the 2-DOF parallel structure is partially decoupled to obtain simple forward/inverse kinematic solutions in which a closed-loop subchain "RPRR" is included. The 7-DOF manipulator is then designed, and its third joint axis goes through the spherical center to obtain closed-form inverse kinematic computation. The analytical inverse kinematic solution is drawn by constructing self-motion manifolds. Finally, a physical prototype is developed, and the kinematic analysis is validated via numerical simulation and test results.
\end{abstract}

Keywords hydraulic manipulator, inverse kinematic,

Received January 28, 2021; accepted May 26, 2021

Min CHENG ( $\triangle)$, Zenan HAN

State Key Laboratory of Mechanical Transmissions, Chongqing University, Chongqing 400044, China

E-mail: chengmin@cqu.edu.cn

Ruqi DING $(\bowtie)$

Key Laboratory of Conveyance and Equipment (Ministry of Education), East China Jiaotong University, Nanchang 330044, China

E-mail: dingruqi0791@sina.com

Junhui ZHANG, Bing XU

State Key Laboratory of Fluid Power and Mechatronic Systems, Zhejiang University, Hangzhou 310027, China redundant design, spherical wrist

\section{Introduction}

\subsection{Background}

Multi-degree-of-freedom (multi-DOF) robotic manipulators have been widely adopted to replace humans and perform dangerous tasks in hazardous environments. For example, dozens of mobile field robots with dual multiDOF arms developed by different teams worldwide attended the finals of the US Defense Advanced Research Projects Agency Robotics Challenge [1], in which several emergency tasks, such as driving, operating valves, plugging, and switching, were involved. Given the existence of numerous heavy-duty tasks, hydraulic robotic manipulators (HRMs) with complex DOFs have been widely used in mobile areas [2], such as in construction, rescue, and exploration [3-5]. As mobile tasks become more complicated, redundant hydraulic manipulators with 7 DOFs, such as electrically driven redundant robots (e.g., CENTAURO and NimbRo [6]), are increasingly required to improve flexibility and dexterity. For example, Kivelä et al. [7] from Tampere University in Finland designed an 8-DOF hydraulic manipulator for the International Thermonuclear Experimental Reactor and found that its redundancy feature was beneficial for optimizing the execution of remote handling tasks. Liang et al. [8] designed a 7-DOF HRM with seven revolute joints for rescue areas during natural disasters. $\mathrm{Li}$ et al. [9] developed a 7-DOF HRM for industrial manufacturing; this HRM consisted of five revolute joints and two prismatic ones. On the basis of the aforementioned literature, HRM redundancy contributes to solving constraint problems due to complex surrounding environments.

\subsection{Problem statement}

HRMs are typically operated by humans in unstructured 
environments to implement diversified tasks. Kinematic similarity between the slave manipulator and the master arm is an important requirement for achieving masterslave intuitive kinematic operation. Thus, specially designed master joysticks are necessary as functional replicas of slave manipulators [3]. Operation efficiency can be further improved to generate human-like movements of a slave manipulator through anthropomorphic configuration [10]. However, current HRMs have not yet achieved anthropomorphic design, and two major problems exist as follows.

1) Most existing HRMs adopt nonspherical 3-DOF wrists, also called offset wrists [4,5,8,11-14]. In contrast with spherical wrists in which three joint axes intersect at the wrist center, a nonspherical wrist cannot decouple an endpoint's orientation from position, and thus, the inverse kinematic solution cannot be easily obtained. With regard to electrically driven robots, hydraulic roll-pitch-roll (RPR) spherical wrists have been developed recently for nuclear [15] or underwater [16] applications by using three rotary actuators. However, the RPR wrist also exhibits a different layout from that of human wrist, which adopts a roll-pitch-yaw (RPY) structure [17]. Moreover, a singularity configuration is observed when two roll axes are collinear at the zero position.

2) Classic redundant anthropomorphic configurations for electrically driven robotics, e.g., sphere-rotationsphere (SRS) [18], are available to achieve analytical inverse kinematic computation; however, they are inappropriate for HRMs due to the difference in driven components. In terms of cost and performance, HRMs use more linear cylinders, while servo rotary motors are the common choice in electrically driven areas. A large installation space is required for hydraulic linear cylinders to generate rotary motion (e.g., boom motion of excavators). Therefore, anthropomorphic HRM configuration should be redesigned rather than directly adopting an electrically driven robot layout.

\subsection{Contributions}

To overcome the aforementioned problems, the contributions of the current study are as follows:

1) An RPY wrist that consists of a parallel yaw/pitch motion plate connected serially to the roll structure is first proposed. Designed as a 1RPRRR-1SPU mechanism ("R", "P", "S", and "U" denote revolute, prismatic, spherical, and universal joints, respectively; the underlined letter indicates the active joint), the 2-DOF parallel structure is decoupled partially to obtain closedform forward/inverse kinematic solutions. Compared with the RPR wrist, one benefit of the RPY wrist is eliminating orientation singularity and increasing similarity to human wrist motion. Another benefit is that two hydraulic linear cylinders with low cost and low friction replace two rotary cylinders with high cost (vane type) or high friction (helical gear type).

2) On the basis of the proposed wrist, a 7-DOF anthropomorphic hydraulic manipulator is designed in which the third joint axis goes through the center of the wrist to simplify kinematic analysis. The analytical inverse kinematic solution is presented via self-motion manifolds to describe redundancy uniquely.

The remainder of this paper is organized as follows. The mechanisms of the spherical wrist and the manipulator are described in Sections 2 and 3, respectively. Section 4 introduces the prototype, numerical verification, and test result. Conclusions are drawn in Section 5.

\section{Design of the spherical wrist}

\subsection{Structural description}

An HRM should be heavy duty and have a compact size to implement complex tasks in unstructured areas. Thus, wrist design has two major concerns as follows:

1) For a spherical wrist, the frame and moving linkages should be sufficiently strong under biaxial loading conditions (i.e., pull or push). Thus, a tendon/cable drive is infeasible for a humanoid wrist due to the large torque demand and heavy load conditions. In addition, all kinematic pairs should be carefully selected to ensure load capacity and motion range.

2) A spherical wrist should be as compact as possible. An epicyclical gear train is a poor choice due to its large size for heavy loads, although it has been widely used in electrically driven wrists. Solutions that use more pairs and links are inappropriate, e.g., the humanoid wrist in Ref. [19] that included 10 joints and 10 moving links.

Given the two aforementioned concerns, the push-rod mechanism is considered an appropriate transmission approach. In contrast with electrically actuated areas, the use of a pure 3-DOF parallel wrist [20] is limited in hydraulic areas because it is too large to be installed on a serial manipulator, particularly in compact environments. As shown in Fig. 1, we adopt a hybrid serial-parallel concept inspired by the human forearm, which uses the radius and the ulna to achieve wrist deviation, to design the wrist as a revolute-universal chain. Two parallel linear cylinders are used to achieve pitch/yaw motion. The detailed wrist structure is then designed as shown in Figs. 1 and 2.

Points $A$ and $B$ denote the kinematic pair centers of the pitch cylinder, while Points $C$ and $D$ denote the kinematic pair centers of the yaw cylinder. The spherical RPY wrist is driven by two parallel linear cylinders for the pitch/yaw motion and a rotary hydraulic cylinder for the roll motion. The pitch cylinder is connected to the base frame and the cross shaft by two revolute pairs. The yaw cylinder is connected to the base frame by a spherical pair and to a 


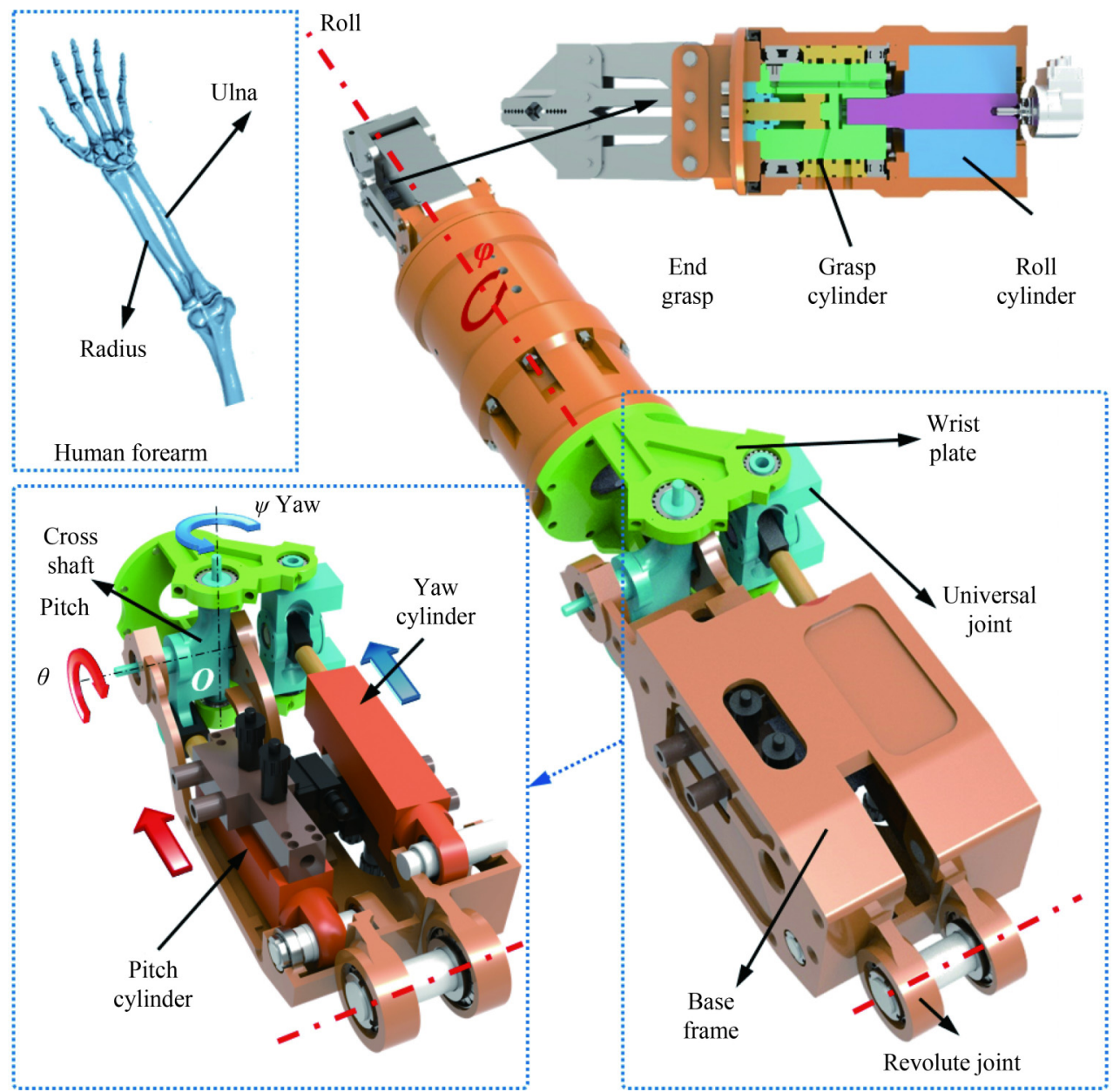

Fig. 1 Structure of the RPY spherical wrist.

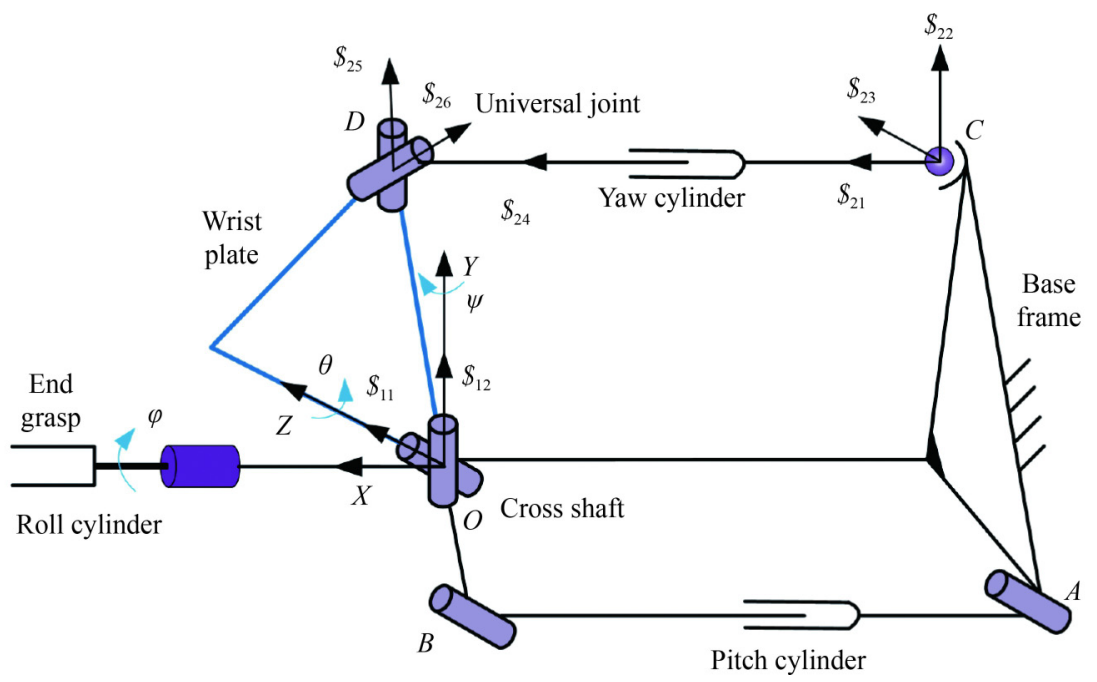

Fig. 2 Kinematic diagram of the RPY spherical wrist.

wrist plate via a universal joint. Moreover, the cross shaft is connected to the base frame via a revolute pair to construct a closed-loop subchain with a four-bar linkage. In addition, the cross shaft is connected to the wrist plate via a revolute pair. In Fig. 1 , Point $D$ is on the $O Z$ axis and Point $B$ is on the $Y O Z$ plane at the zero position.

The two yaw/pitch cylinder pairs are considered two prismatic pairs because two local freedoms of the 
cylinders rotate about their own axes. The pitch/yaw structure is considered a 1RPRRR-1S $\underline{P} U$ asymmetrical mechanism. The upper bracket indicates the presence of a closed-loop subchain, including the "RRRR" kinematic pairs, because the cross shaft is connected to the frame. Thus, the wrist center is maintained under different orientations. The two cylinders are placed into the hollow frame that bears and transmits the exerted force from the end effector. The base frame can be fixed onto a serial manipulator via a revolute pair. Extended discussions on the yaw/pitch mechanism are provided as follows:

1) Selection of driven components. Apart from hydraulic linear cylinders, another choice is hydraulic rotary cylinders. However, the 2-DOF parallel mechanism with rotary driven parts (e.g., U-2RRR [21]) evidently occupies a larger radial space, and thus, it is inappropriate for this application. One benefit of the proposed design is that two linear servo cylinders with low cost and low friction replace two rotary cylinders in RPR wrists with high cost (vane type) or high friction (helical spline type).

2) Selection of kinematic pairs. The spherical pair should be carefully selected because its motion range and load capacity (particularly retracting load) are limited in this case. Therefore, solutions that require spherical joints with a large motion range and high bearing capacity, such as the fully decoupled parallel wrist in Ref. [22] (included four "S" pairs) and U-2PUS [23], are unsuitable.

3) Utilization of passive limbs. 2-DOF parallel mechanisms that use passive limbs to improve stiffness, such as 2SPR-SR [24] and 2UPR-SS [25], have been proposed. However, the radial dimension of the wrist should be as small as possible, and thus, adding a moving limb is difficult to avoid motion interference. The wrist is extremely compact; hence, structure flexibility can be disregarded, particularly when installed on a serial hydraulic arm with weak rigidity.

4) Numbers of pairs and linkages. To achieve 2-DOF yaw/pitch motion, smaller numbers of kinematic pairs and linkages $(n=6$ and $g=7)$ are selected for the U-2PUS [24] or U-2PSU [26] mechanism. However, assigning the universal/spherical joints and achieving trade-off among compactness, workspace, and torque are difficult. A closed-loop subchain is designed by considering this point, and only one "U" pair and one "S" pair are necessary.

The only rotary cylinder is used to drive the roll joint connected serially to the wrist plate, such that the roll motion is decoupled from the yaw/pitch motion. The axis of the roll cylinder is aligned to the spherical center. Either a vane cylinder or a helical spline cylinder is available to achieve roll motion. The rack-and-pinion cylinder is limited in this application due to its large installation space and the hydraulic motor with an epicyclic gearbox. To reduce structure size, the roll cylinder and the grasp cylinder are installed in a uniform hollow shell, as shown in Fig. 2. The roll cylinder drives the hydraulic rotary coupling in which the piston rod of the grasp cylinder is installed. The pressurized oil goes across the hydraulic coupling and into the cylinder chamber to realize grasp movement. Kinematic analysis is then conducted to obtain the relationship between cylinder positions and yaw/pitch angles.

\subsection{Mobility analysis}

As shown in Fig. 3, the fixed orthonormal reference frame $\{O-X Y Z\}$ is attached to the wrist, and the original point $O$ coincides with the wrist center. Wrist orientation is represented as three angles $[\theta, \psi, \varphi]^{\mathrm{T}}$ via the Euler angle form. The angles are $[\theta, \psi, \varphi]^{\mathrm{T}}=[0,0,0]^{\mathrm{T}}$ at the zero position. Under the base frame $\{O-X Y Z\}$, the positions of Points $A-F$ is expressed as $\boldsymbol{P}_{A}=\left[x_{A}, y_{A}, z_{A}\right]^{\mathrm{T}}$, $\boldsymbol{P}_{B}=\left[b \sin \theta,-b \cos \theta, z_{B}\right]^{\mathrm{T}}, \quad \boldsymbol{P}_{C}=\left[x_{C}, 0, z_{C}\right]^{\mathrm{T}}, \quad \boldsymbol{P}_{D}=$ $[d \sin \psi \cos \theta, d \sin \psi \sin \theta, d \cos \psi]^{\mathrm{T}}, \quad \boldsymbol{P}_{E}=\left[0,0, z_{E}\right]^{\mathrm{T}}, \quad$ and $\boldsymbol{P}_{F}=[-f \sin \theta, f \cos \theta, 0]^{\mathrm{T}}$. The positions of Points $A, C$, and $E$ are constant relative to the reference frame $\{O-X Y Z\}$, whereas the positions of Points $B, D$, and $F$ are variant. The geometrics are defined as $\|\overrightarrow{B E}\|=b$, $\|\overrightarrow{O D}\|=d$, and $\|\overrightarrow{O F}\|=f$.

A closed-loop subchain RPRR with four-bar linkages exists, and thus, the pitch/yaw 1RPRRR-1SPU mechanism can be equivalent to the 1 RR-1SPU mechanism. Then, screw-based theory is used to analyze the DOF number of the parallel mechanism. For the equivalent 1RR-1SPU mechanism shown in Fig. 2, the kinematic screw system of the branch chain " 1 RR" under the base frame is given by

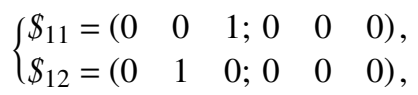

where $\$_{i j}$ is the unit screw of the $i$ th joint in the $j$ th limb. The corresponding reciprocal screw system can then be represented by

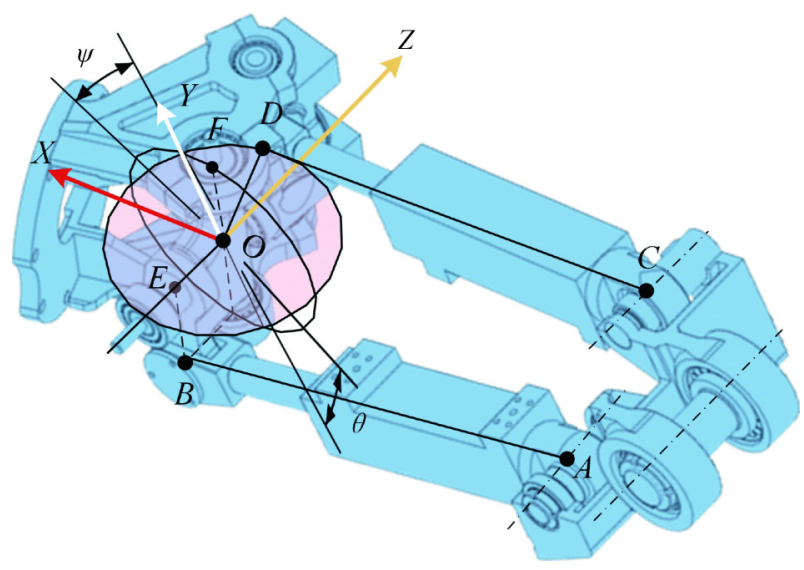

Fig. 3 Principle of the pitch/yaw motion mechanism. 


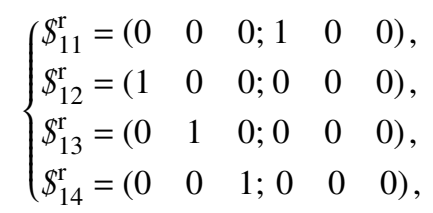

where $\$_{i j}^{\mathrm{r}}$ is the reciprocal screw of the $i$ th joint in the $j$ th limb. In addition, the kinematic screw system of the branch chain " $1 \mathrm{SP} U$ " is given by

$$
\left\{\begin{array}{l}
\$_{21}=\left(\begin{array}{lllll}
1 & 0 & 0 ; 0 & z_{C} & 0
\end{array}\right) \\
\$_{22}=\left(\begin{array}{lllll}
0 & 1 & 0 ;-z_{C} & 0 & x_{C}
\end{array}\right) \\
\$_{23}=\left(\begin{array}{lllll}
0 & 0 & 1 ; 0 & -x_{C} & 0
\end{array}\right) \\
\$_{24}=\left(\begin{array}{lllll}
0 & 0 & 0 ;-x_{C} & 0 & d-z_{C}
\end{array}\right) \\
\$_{25}=\left(\begin{array}{lllll}
0 & 1 & 0 ;-d & 0 & 0
\end{array}\right) \\
\$_{26}=\left(\begin{array}{lllll}
d-z_{C} & 0 & x_{C} ; 0 & d^{2}-d z_{C} & 0
\end{array}\right)
\end{array}\right.
$$

No corresponding reciprocal screw exists for the branch chain "1SPU". From Eqs. (1)-(3), no common and parallel redundancy constraints are found, indicating that $\lambda=0$ and $v=0$ in Eq. (4), where $\lambda$ is the number of common constraint and $v$ is the number of redundancy constraint. Moreover, the number of links is $n=5$ for the 1RR-1SPU mechanism; and the number of joints is $g=5$, including one spherical joint, one universal pair, one prismatic pair, and two revolute joints. Thus, the DOF of the equivalent mechanism $(M)$ can be calculated as [27]

$$
\begin{aligned}
M & =(6-\lambda)(n-g-1)+\sum_{j=1}^{g} f_{j}+v \\
& =(6-0)(5-5-1)+(1 \times 3+1 \times 2+2 \times 1+2 \times 1)+0=2,
\end{aligned}
$$

where $f_{i}$ is the DOF of joints.

\subsection{Kinematic analysis}

For the proposed wrist, kinematic analysis involves transferring the task space to the joint space and then to the cylinder space. That is, the objective of the inverse kinematic analysis is to determine cylinder displacements under a set of specified Euler angles. The vectors $\overrightarrow{A B}$ and $\overrightarrow{C D}$ are expressed as

$$
\overrightarrow{A B}=\left[b \sin \theta-x_{A},-b \cos \theta-y_{A}, z_{B}-z_{A}\right]^{\mathrm{T}},
$$

$$
\overrightarrow{C D}=\left[d \sin \psi \cos \theta-x_{C}, d \sin \psi \sin \theta, d \cos \psi-z_{C}\right]^{\mathrm{T}} .
$$

Evidently, $\|\overrightarrow{A B}\|$ and $\|\overrightarrow{C D}\|$ can be derived by

$\|\overrightarrow{A B}\|=l_{0 \mathrm{p}}+x_{\mathrm{p}}=\sqrt{\left(b \sin \theta-x_{A}\right)^{2}+\left(b \cos \theta+y_{A}\right)^{2}+\left(z_{B}-z_{A}\right)^{2}}$,

$$
\begin{aligned}
\|\overrightarrow{C D}\| & =l_{0 \mathrm{y}}+x_{\mathrm{y}} \\
& =\sqrt{\left(d \sin \psi \cos \theta-x_{C}\right)^{2}+d^{2} \sin ^{2} \psi \sin ^{2} \theta+\left(d \cos \psi-z_{C}\right)^{2}},
\end{aligned}
$$

where $l_{0 \mathrm{p}}$ is the minimum pitch cylinder length, $x_{\mathrm{p}}$ is the pitch cylinder displacement, $l_{0 y}$ is the minimum yaw cylinder length, and $x_{\mathrm{y}}$ is the yaw cylinder displacement. The yaw angle depends on $x_{\mathrm{p}}$ and $x_{\mathrm{y}}$, but the pitch angle is only related to $x_{\mathrm{p}}$. Thus, the 2-DOF wrist mechanism is partially decoupled due to the closed-loop chain of the four-bar linkage. The following expression is obtained from Eq. (7):

$$
y_{A} \cos \theta-x_{A} \sin \theta=\frac{\left(l_{0 \mathrm{p}}+x_{\mathrm{p}}\right)^{2}-\left(z_{B}-z_{A}\right)^{2}-b^{2}-x_{A}^{2}-y_{A}^{2}}{2 b} .
$$

Given the pitch cylinder displacement, the pitch angle $\theta$ can be solved on the basis of the actual motion range to derive a feasible solution. Similarly, the following expression is derived from Eq. (8):

$$
z_{C} \cos \psi+x_{C} \sin \psi \cos \theta=-\frac{\left(l_{0 \mathrm{y}}+x_{\mathrm{y}}\right)^{2}-x_{C}^{2}-z_{C}^{2}-d^{2}}{2 d} .
$$

The yaw angle $\psi$ can be obtained on the basis of the yaw cylinder displacement $x_{\mathrm{y}}$ and the pitch angle $\theta$. The forward kinematic problem is generally complex for a parallel structure, but it can be determined easily for the wrist through Eqs. (5)-(10). Moreover, the roll motion is decoupled from the pitch/roll motion, and thus, the roll cylinder position can be directly attained from the roll angle. The geometrical parameters of the wrist can then be selected by considering motion workspace and possible interference.

\subsection{Singularity analysis}

The joint singularity of a general RPY wrist was discussed in Ref. [28], and it can be avoided through joint constraints. Thus, we mostly discuss the singularity of the 1RPRRR-1SPU parallel mechanism. The relationship between cylinder velocity ( $\dot{x}_{\mathrm{p}}$ and $\dot{x}_{\mathrm{y}}$ ) and joint angular velocity $(\dot{\theta}$ and $\dot{\psi})$ can be derived from Eqs. (9) and (10) as

$$
\boldsymbol{J}_{\boldsymbol{x}}\left[\begin{array}{c}
\dot{x}_{\mathrm{p}} \\
\dot{x}_{\mathrm{y}}
\end{array}\right]-\boldsymbol{G}\left[\begin{array}{c}
\dot{\theta} \\
\dot{\psi}
\end{array}\right]=0
$$

where the intermediate matrix of the cylinder displacements $\boldsymbol{J}_{\boldsymbol{x}}=\left[\begin{array}{cc}J_{x 1} & 0 \\ 0 & J_{x 2}\end{array}\right]$, the intermediate matrix of the joint angles $\boldsymbol{G}=\left[\begin{array}{ll}G_{11} & G_{12} \\ G_{21} & G_{22}\end{array}\right]$, and

$$
\begin{gathered}
\left\{\begin{array}{l}
J_{x 1}=\frac{l_{0 \mathrm{p}}+x_{\mathrm{p}}}{b}, \\
J_{x 2}=\frac{l_{0 \mathrm{p}}+x_{\mathrm{y}}}{b},
\end{array}\right. \\
\left\{\begin{array}{l}
G_{11}=-y_{A} \sin \theta-x_{A} \cos \theta, \\
G_{12}=0, \\
G_{21}=-x_{C} \sin \psi \sin \theta, \\
G_{22}=-z_{C} \sin \psi+x_{C} \cos \psi \cos \theta .
\end{array}\right.
\end{gathered}
$$


From Eq. (12), $\left|\boldsymbol{J}_{\boldsymbol{x}}\right| \neq 0$. From Eq. (13), two singularity conditions exist: $G_{11}=0$ and $G_{22}=0$. Then, the corresponding joint angles can be obtained as

$$
\begin{gathered}
G_{11}=0 \rightarrow \tan \theta=-\frac{x_{A}}{y_{A}}, \\
G_{22}=0 \rightarrow \tan \psi=\frac{x_{C} \cos \theta}{z_{C}} .
\end{gathered}
$$

\section{Design of the overall manipulator}

\subsection{Structural design}

A 7-DOF anthropomorphic redundant hydraulic manipulator is then designed on the basis of the proposed RPY wrist. In addition to wrist $\mathrm{pitch} / \mathrm{yaw} / \mathrm{roll}$, the other 4 DOFs for a human arm are shoulder pitch/yaw/roll and elbow pitch. To ensure the motion range, the first joint is determined as a revolute one. The movement of the second joint is typically achieved via a linear hydraulically driven component, such as the boom cylinder of an excavator's arm. Thus, the basic configuration is presented in Fig. 4(a), in which the wrist joints are considered the fifth, sixth, and seventh joints $\left(R_{5}, R_{6}\right.$, and $R_{7}$ ). For the human arm layout, two configurations of Joints $2-4\left(R_{2}, R_{3}\right.$, and $\left.R_{4}\right)$ are selected as shown in Figs. 4 (b) and 4(c). A typical SRS $\left(R_{1}, R_{2}\right.$, and $\left.R_{3}\right)$ redundant structure is formed for the configuration in Fig. 4(b), which has been extensively applied to electrically driven areas, but makes large installation space and unnecessary axial length difficult to avoid. Comparatively, the HRM shown in Fig. 4(c) is more similar to a human arm. This HRM and its $3 \mathrm{D}$ model are designed through this configuration, as shown in Fig. 5. The axis of the third joint goes through the wrist center to conduct self-motion manifolds of the fourth joint easily. A small offset between the first and second joints is allowed and assigned for layout consideration. The first, third, and seventh joints are driven by hydraulic rotary cylinders, while the others are driven by hydraulic linear cylinders. The first joint can be also driven by a high-speed hydraulic motor with a gearbox due to its large installation space.

\subsection{Forward kinematic analysis of the manipulator}

The forward kinematic analysis of the HRM aims to obtain the motion trajectory of the endpoint by transforming the cylinder space into the joint space and then the task space. Under the base frame of the entire HRM, the position and orientation of the endpoint $\boldsymbol{P}_{\mathrm{e}}$ are expressed as

$$
\begin{gathered}
{ }^{0} \boldsymbol{P}_{\mathrm{e}}={ }_{7}^{0} \boldsymbol{T}^{7} \boldsymbol{P}_{\mathrm{e}}, \\
{ }_{7}^{0} \boldsymbol{T}={ }_{1}^{0} \boldsymbol{T}_{2}^{1} \boldsymbol{T}_{3}^{2} \boldsymbol{T}_{4}^{3} \boldsymbol{T}_{5}^{4} \boldsymbol{T}_{6}^{5} \boldsymbol{T}_{7}^{6} \boldsymbol{T} .
\end{gathered}
$$

The transformation matrices $\left({ }_{i}^{i-1} \boldsymbol{T}\right)$ are given by

$$
\begin{gathered}
{ }_{i}^{i-1} \boldsymbol{T}=\left[\begin{array}{cccc}
\cos q_{i} & -\sin q_{i} \cos \alpha_{i} & \sin q_{i} \sin \alpha_{i} & a_{i} \cos q_{i} \\
\sin q_{i} & \cos q_{i} \cos \alpha_{i} & -\cos q_{i} \sin \alpha_{i} & a_{i} \sin q_{i} \\
0 & \sin \alpha_{i} & \cos \alpha_{i} & d_{i} \\
0 & 0 & 0 & 1
\end{array}\right], \\
i=1,2, \ldots, 7, \\
{ }^{7} \boldsymbol{P}_{\mathrm{e}}=\left[\begin{array}{cccc}
1 & 0 & 0 & 0 \\
0 & 1 & 0 & 0 \\
0 & 0 & 1 & l_{\mathrm{e}} \\
0 & 0 & 0 & 1
\end{array}\right],
\end{gathered}
$$

where $q_{i}$ is the joint angle of the manipulator, $\alpha_{i}$ is the link wrist angle between two adjacent joints, $a_{i}$ is the link length between two adjacent joints, and $d_{i}$ is the link offset of the joint $(i=1,2, \ldots, 7)$, and $l_{\mathrm{e}}$ is the distance from the wrist center to the endpoint of the manipulator. The coordinate $O_{i}$ of the $i$ th joint is denoted as $\left[x_{i}, y_{i}, z_{i}\right]^{\mathrm{T}}$ under the base frame of the manipulator; and $O_{5}, O_{6}$, and $\mathrm{O}_{7}$ coincide with one another. The position of the end effector is defined as $\left[x_{\mathrm{e}}, y_{\mathrm{e}}, z_{\mathrm{e}}\right]^{\mathrm{T}}$.

\subsection{Inverse kinematic of the manipulator}

The solutions for the inverse kinematic problem of serial redundant manipulators can be categorized into positionand velocity-based inverse methods. The latter exhibits the disadvantages of expensive computation and difficulty to map joint limits. From the Pieper criteria

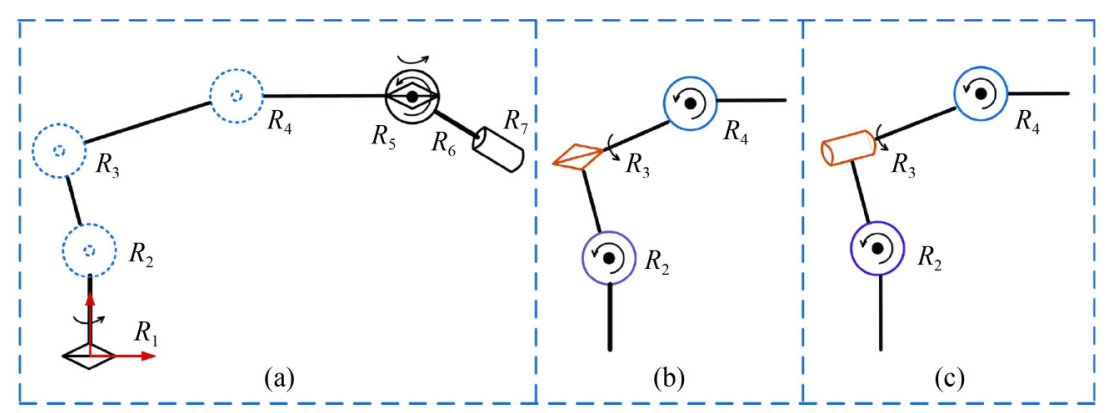

Fig. 4 Selected configurations of the 7-DOF HRM. (a) Basic configuration, (b) SRS configuration, and (c) selected configuration. 


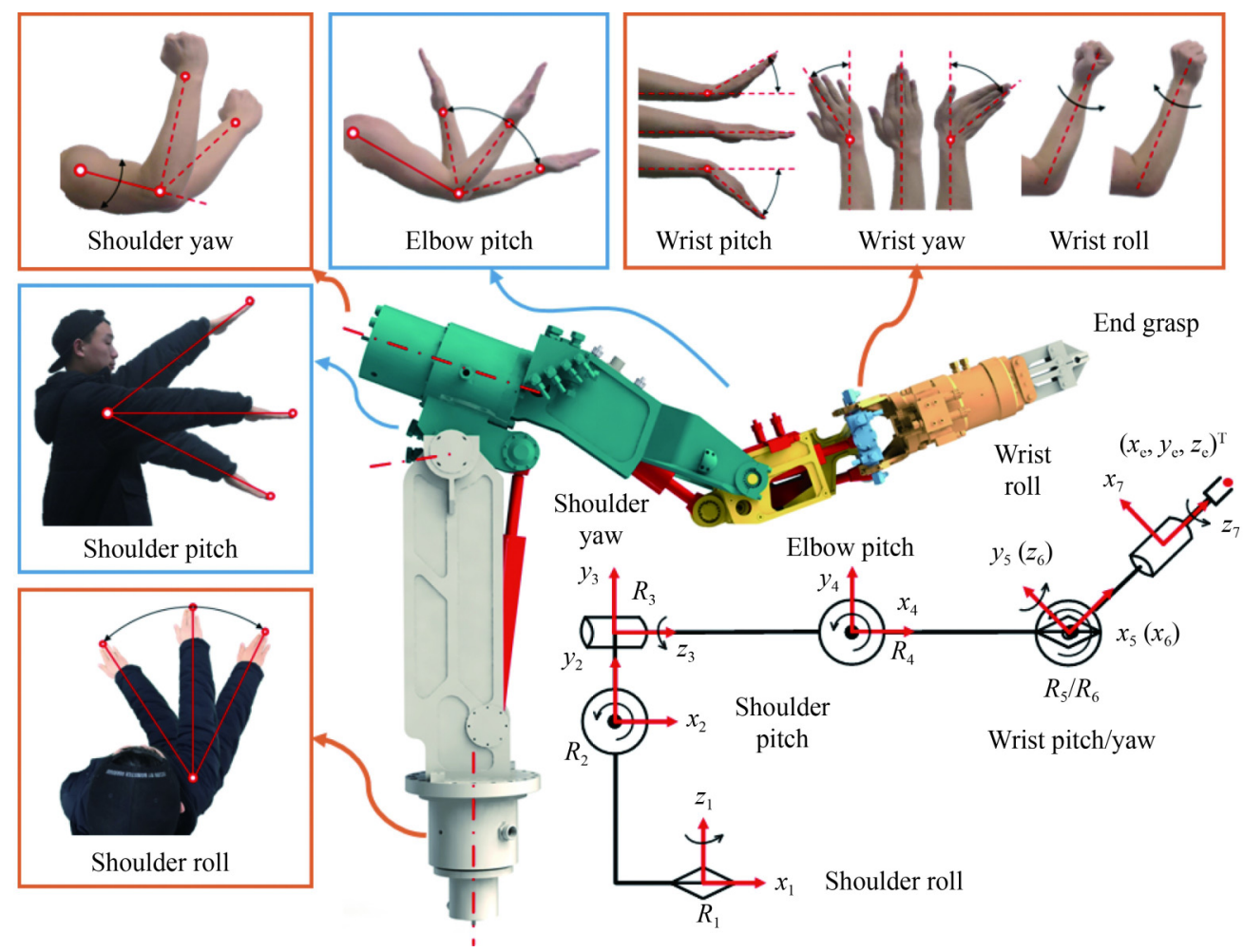

Fig. 5 Configuration and 3D model of the proposed HRM.

[29], the analytical inverse kinematic solution of a 6-DOF arm with a spherical wrist is simple to obtain. Thus, a position-based inverse kinematic solution for the designed HRM introduces the joint parameterization method, but it demonstrates the deficiency that redundancy cannot be described uniquely. Point $O_{4}$ is on the rotation axis of Joint 3 , whose position does not change under different joint angles $q_{3}$. Therefore, we construct self-motion manifolds to obtain the inverse kinematic solution. The feasible positions of the elbow joint can be defined by a redundancy curve resulting from the intersection of the ellipsoid Eq. (20) and the sphere Eq. (21):

$$
\begin{gathered}
\frac{\left(\sqrt{x_{4}^{2}+y_{4}^{2}}-a_{1}\right)^{2}}{a_{2}^{2}+d_{3}^{2}}+\frac{\left(z_{4}-d_{1}\right)^{2}}{a_{2}^{2}+d_{3}^{2}}=1, \\
\frac{\left(x_{5}-x_{4}\right)^{2}}{a_{4}^{2}}+\frac{\left(y_{5}-y_{4}\right)^{2}}{a_{4}^{2}}+\frac{\left(z_{5}-z_{4}\right)^{2}}{a_{4}^{2}}=1 .
\end{gathered}
$$

The redundancy curve can be obtained by combining the intersections for all the values of $z_{4}$ in the interval $\left[z_{4 l o w}, z_{4 \text { upp }}\right]$, where $z_{4 l o w}$ and $z_{4 \text { upp }}$ are the lower and upper bounds of the $z$-coordinate of elbow joint $R_{4}$, respectively. Correspondingly, the following steps are performed to obtain the inverse kinematic solution.

1) Step 1: The coordinate of Point $O_{5}$ is calculated for a specific endpoint ${ }^{0} \boldsymbol{P}_{\mathrm{e}}$. The transformation matrix is given by

$$
{ }_{7}^{0} \boldsymbol{T}=\left[\begin{array}{cccc}
* & * & * & x_{5} \\
* & * & * & y_{5} \\
* & * & * & z_{5} \\
0 & 0 & 0 & 1
\end{array}\right]={ }^{0} \boldsymbol{P}_{\mathrm{e}}\left({ }^{7} \boldsymbol{P}_{\mathrm{e}}\right)^{-1} .
$$

Thus, the coordinate of Point $O_{5}=\left[x_{5}, y_{5}, z_{5}\right]^{\mathrm{T}}$ can be obtained from Eq. (22). The symbol “*” denotes the omitted elements, which are not required in the calculation step.

2) Step 2: The range of $z_{4}$ is calculated. In accordance with the HRM configuration, the following expressions are derived:

$$
\left\{\begin{array}{l}
z_{4}\left(q_{2}\right)=a_{2} \cos q_{2}+d_{3} \sin q_{2}+d_{1} \\
z_{4} \in\left[z_{5}-a_{4}, z_{5}+a_{4}\right]
\end{array}\right.
$$

Then, the following is defined:

$$
\left\{\begin{array}{l}
z_{4 \text { low }}=\max \left[\underset{q_{2 \min } \leqslant q_{2} \leqslant q_{2 \max }}{\arg \min } z_{4}\left(q_{2}\right), z_{5}-a_{4}\right], \\
z_{4 \text { upp }}=\min \left[\underset{q_{2 \min } \leqslant q_{2} \leqslant q_{2 \max }}{\arg \max } z_{4}\left(q_{2}\right), z_{5}+a_{4}\right] .
\end{array}\right.
$$

3) Step 3: For a specified $z_{4}$ bounded by Eq. (24), the joint angles $q_{1}-q_{4}$ can be solved on the basis of the following expressions:

$$
\begin{gathered}
q_{1}=-\operatorname{atan} \frac{x_{4}}{y_{4}} \in\left[q_{1 \min }, q_{1 \max }\right], \\
q_{2}=\arcsin \frac{z_{4}-d_{1}}{\sqrt{a_{2}^{2}+d_{3}^{2}}}-\operatorname{atan} \frac{a_{2}}{d_{3}} \in\left[q_{2 \min }, q_{2 \max }\right],
\end{gathered}
$$




$$
\begin{gathered}
q_{4}=\arccos \frac{\overrightarrow{O_{3} O_{4}} \cdot \overrightarrow{O_{4} O_{5}}}{\left|\overrightarrow{O_{3} O_{4}}\right| \cdot\left|\overrightarrow{O_{4} O_{5}}\right|} \in\left[q_{4 \min }, q_{4 \max }\right], \\
q_{3}=\arcsin \frac{x_{5} \cos q_{1}+y_{5} \sin q_{1}}{a_{4} \sin q_{4}} \in\left[q_{3 \min }, q_{3 \mathrm{max}}\right] .
\end{gathered}
$$

4) Step 4: When the position and orientation of Point $\mathrm{O}_{4}$ are determined, the joint angles $q_{5}-q_{7}$ are calculated. The transformation matrix ${ }_{7}^{4} \boldsymbol{T}$ for the wrist is given by

$$
\begin{aligned}
{ }_{7}^{4} \boldsymbol{T} & =\left({ }_{4}^{0} \boldsymbol{T}\right)^{-1}{ }^{0} \boldsymbol{P}_{\mathrm{e}}\left({ }^{7} \boldsymbol{P}_{\mathrm{e}}\right)^{-1}=\left[\begin{array}{ccc}
a_{11} & a_{12} & a_{13} \\
a_{21} & a_{22} & a_{23} \\
a_{31} & a_{32} & a_{33}
\end{array}\right] \\
& =\left[\begin{array}{ccc}
* & * & \cos q_{5} \cos q_{6} \\
* & * & \sin q_{5} \cos q_{6} \\
-\cos q_{6} \cos q_{7} & \cos q_{6} \sin q_{7} & -\sin q_{6}
\end{array}\right] .
\end{aligned}
$$

The joint angles $q_{5}-q_{7}$ can then be calculated by

$$
\begin{gathered}
q_{6}=-\arcsin a_{33}, \\
q_{5}=\operatorname{atan} 2\left(a_{23}, a_{13}\right), \\
q_{7}=-\operatorname{atan} 2\left(a_{32}, a_{31}\right) .
\end{gathered}
$$

In summary, the seven joint angles of the HRM are determined in sequence if a specified position and orientation of the endpoint are given.

\section{Case study and validation}

\subsection{Prototype of the manipulator}

The manipulator prototype shown in Fig. 6 was developed. It was equipped with a compact grasp structure to implement dexterous tasks in rescue or other hazardous applications. The hydraulic servo valves were installed on the manipulator to improve the natural frequency of the hydraulic joint. Hydraulic rotary couplings were utilized to import the pressurized oil from the anterior joint. Absolute optical encoders were used to capture the joint angles. The maximum payload and the maximum reach radius of the manipulator were $500 \mathrm{~kg}$ and $1.976 \mathrm{~m}$, respectively. The strength of the structural frame was also checked successfully under the maximum load by using finite element analysis.

The DH parameters are provided in Table 1. The geometrical parameters of the spherical wrist were selected as follows: $x_{A}=-0.41 \mathrm{~m}, y_{A}=-0.07 \mathrm{~m}, z_{A}=z_{B}=$ $z_{E}=-0.052 \mathrm{~m}, x_{C}=-0.411 \mathrm{~m}, z_{C}=0.07 \mathrm{~m}, l_{0 \mathrm{p}}=0.343 \mathrm{~m}$, $l_{0 \mathrm{y}}=0.35 \mathrm{~m}, x_{\mathrm{p}} \in[0,0.134 \mathrm{~m}], x_{\mathrm{y}} \in[0,0.126 \mathrm{~m}], l_{\mathrm{e}}=0.741$ $\mathrm{m}$, and $b=d=f=0.1 \mathrm{~m}$. On the basis of Eq. (9), pitch range was calculated as $\left[-42.09^{\circ}, 42.05^{\circ}\right]$. The yaw angles under different positions of the pitch and the yaw cylinders are shown in Fig. 7. The limitations of the yaw angles under different pitch angles are calculated as
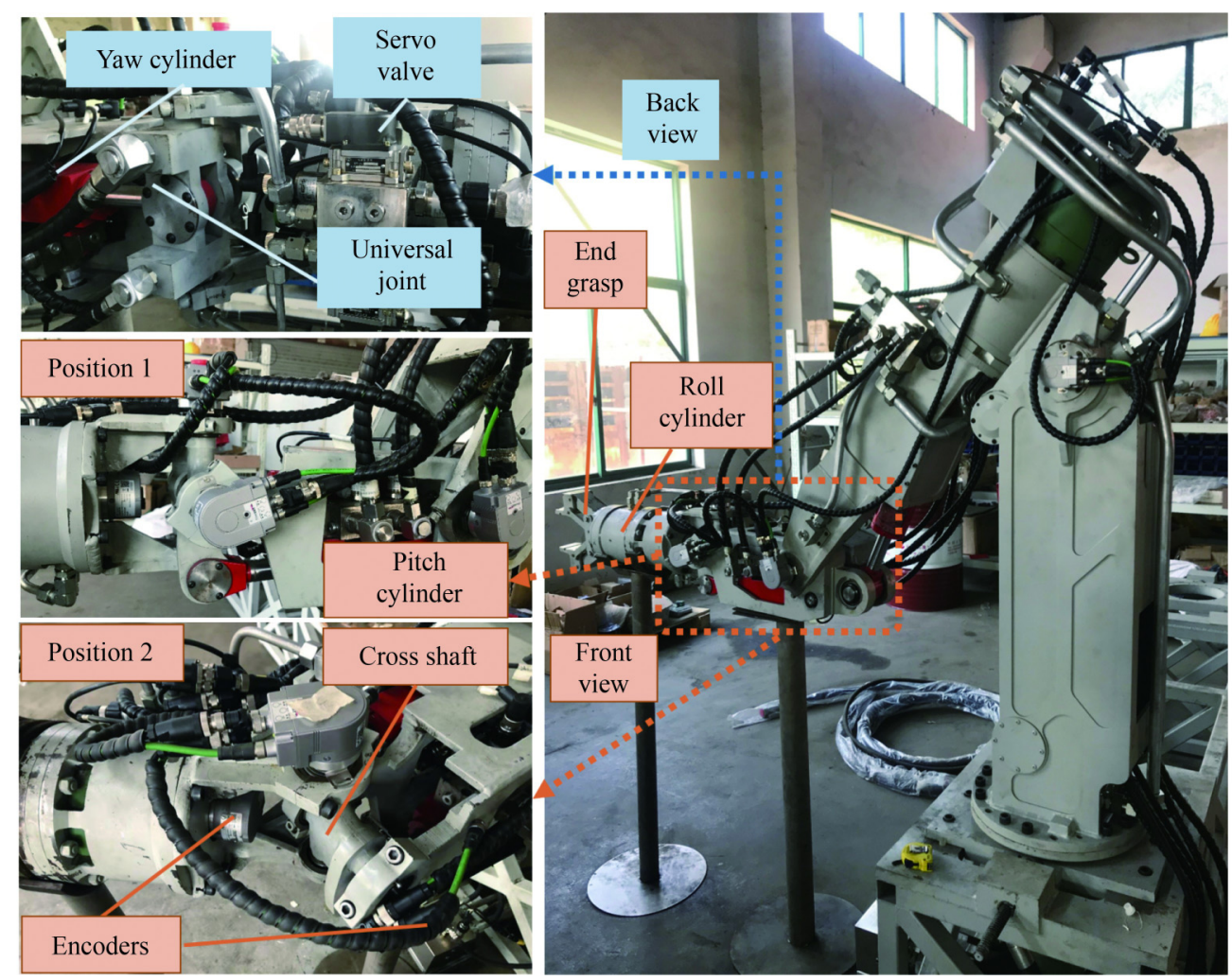

Fig. 6 Prototype of the spherical wrist and manipulator. 
Table 1 DH parameters of the manipulator

\begin{tabular}{lccccc}
\hline$i$ & $q_{i} /\left(^{\circ}\right)$ & $a_{i} / \mathrm{m}$ & $\alpha_{i} /\left(^{\circ}\right)$ & $d_{i} / \mathrm{m}$ & Joint angle range $/\left(^{\circ}\right)$ \\
\hline 1 & $q_{1}-90$ & -0.055 & -90 & 1.015 & {$[-45,45]$} \\
2 & $q_{2}-90$ & 0.225 & 90 & 0 & {$[-80,42]$} \\
3 & $q_{3}$ & 0 & -90 & 0.846 & {$[-45,45]$} \\
4 & $q_{4}-90$ & 0.360 & 0 & 0 & $0,124]$ \\
5 & $q_{5}$ & 0 & -90 & 0 & Given in Fig. 8 \\
6 & $q_{6}+90$ & 0 & 0 & 0 & Given in Fig. 8 \\
7 & $q_{7}$ & 0 & & {$[-135,135]$} \\
\hline
\end{tabular}

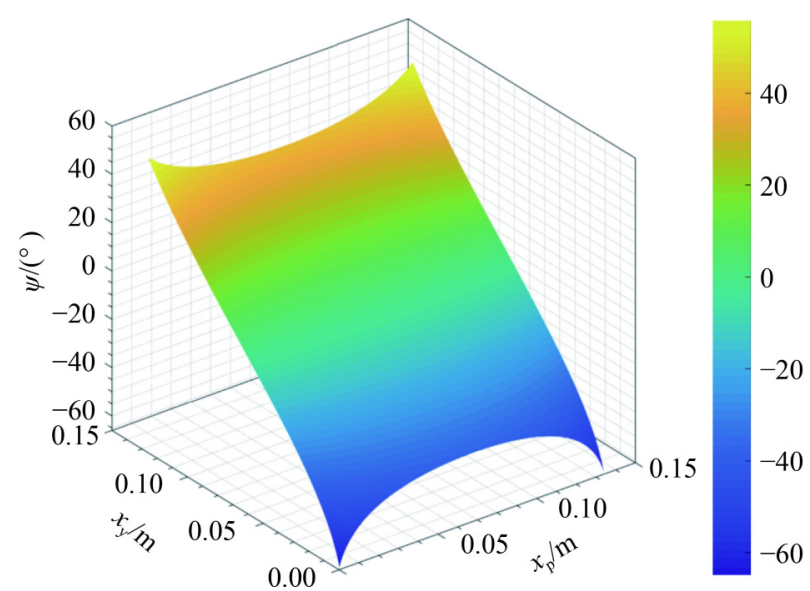

Fig. 7 Yaw angles under different pitch/yaw cylinder displacements.

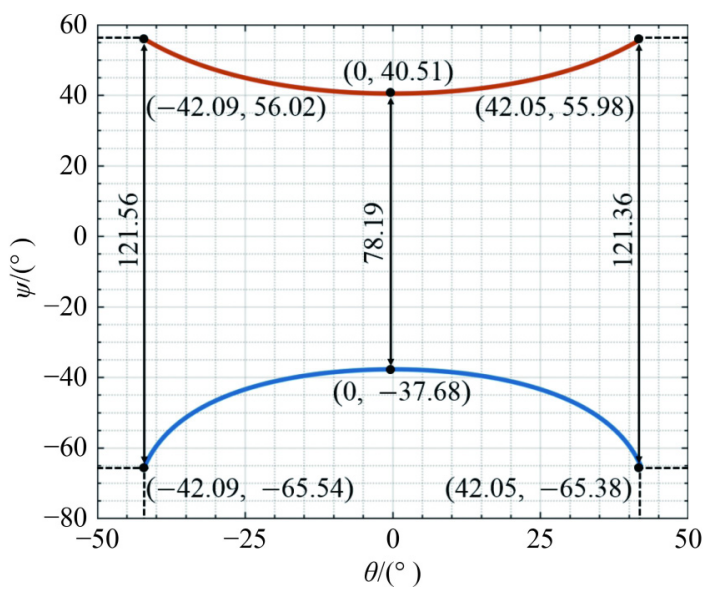

Fig. 8 Yaw limitations under different pitch angles.

shown in Fig. 8. The yaw motion range was smaller when the pitch angle was near zero. The minimum yaw range was $\left[-37.68^{\circ}, 40.51^{\circ}\right]$ at $\theta=0^{\circ}$, while the maximum range was $\left[-65.54^{\circ}, 56.02^{\circ}\right]$ at $\theta=-42.09^{\circ}$. In addition, the roll angle was controlled separately by the roll cylinder. The first singularity condition of the wrist that satisfies Eq. (14) is depicted in Fig. 9(a). Two singular configurations occurred when Point $B$ was located at $B_{1}$ or $B_{2}$. As illustrated in Fig. 9(b), two more singular configurations were observed when Point $D$ was located at $D_{1}$ or $D_{2}$, for which Eq. (15) was satisfied. Figure 9(c) shows the singular yaw angles under different pitch angles in this case. Considering the motion range of the wrist, singular configurations cannot be achieved. Moreover, the condition number $\kappa \in[1, \infty)$, which is defined as the ratio of the maximum and minimum singular values of the Jacobian matrix [30], was introduced to evaluate the dexterity performance of the wrist mechanism. The Jacobian matrix was then calculated as the motion at the end effector with respect to the wrist center on the basis of the motion range. The calculated result is presented in Fig. 10, and it falls within the range of $[1.24,3.58]$. Good dexterity performance can be obtained within the wrist's workspace. Thereafter, numerical simulation and experimental tests were conducted to verify the kinematic analysis of the physical prototype.

\subsection{Simulation verification of the kinematic analysis}

First, four ideal boundary trajectories of the endpoint on different $X O Z$ planes were calculated using the Monte Carlo method, as shown in Fig. 11. Then, 1000 sampling points were selected to verify the analytical inverse kinematic solution through numerical simulation. Position errors between the 1000 calculated and selected points are shown in Fig. 12 from the ideal boundary trajectories. The position errors along the $X, Y$, and $Z$ axes were all smaller than $1.0 \times 10^{-11} \mathrm{~mm}$. Such finding was generally caused by floating-point computation. Moreover, the inverse kinematic computation time was nearly $28.5 \mathrm{~ms}$ per thousand times (computer configuration: AMD Ryzen 7 4700U@2.0 GHz and 16 GB RAM). Thus, the proposed inverse computational method exhibits the advantages of high position efficiency and fast calculation time. It can be utilized in the real-time master-slave operation of a hydraulic manipulator in actual applications.

\subsection{Experimental verification of the kinematic analysis}

A total of 100 points were randomly selected from the ideal boundary trajectories in Fig. 11 to verify the 

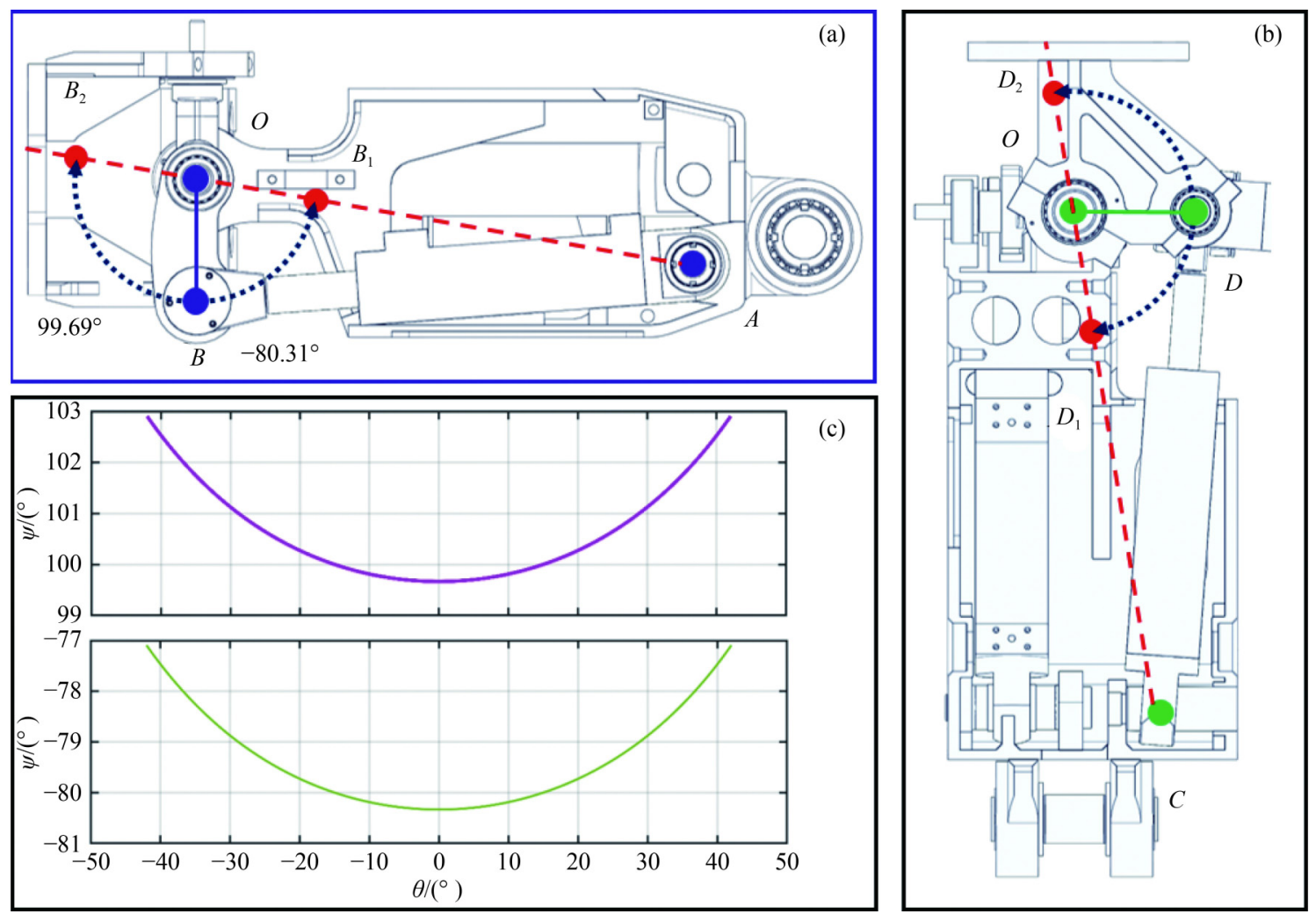

Fig. 9 Singular configurations of the hybrid series-parallel mechanism. (a) Singularity condition satisfying Eq. (14), (b) singularity condition satisfying Eq. (15), and (c) singular yaw angles under different pitch angles.

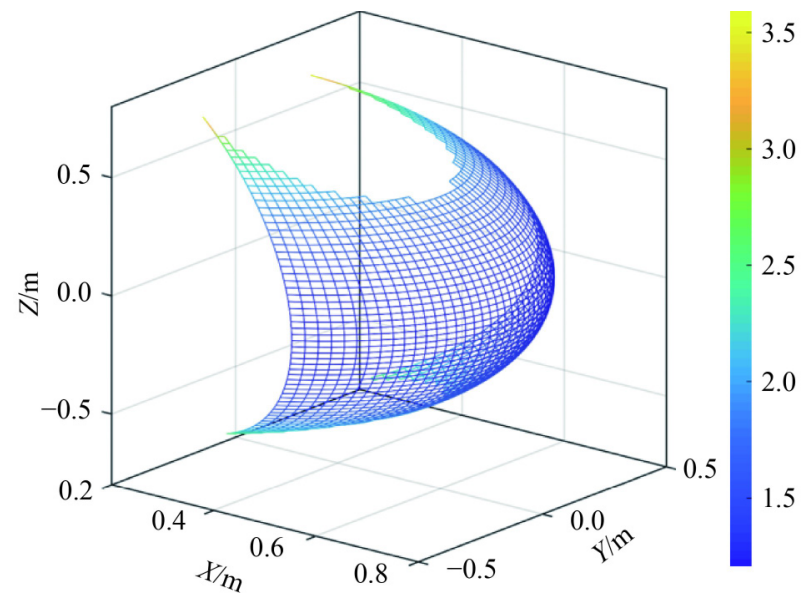

Fig. 10 Dexterity performance of the hybrid series-parallel mechanism.

forward/inverse kinematic computation. Figure 13 shows a laser tracker (API Radian) and a measuring target ball installed on the end effector of the prototype to validate the kinematic analysis. The 100 ideal selected and measurement points that were actually reached by the end effector are presented in Fig. 13, and these points were consistent with one another. As depicted in Fig. 14, the position errors between the ideal and measurement points were smaller than $10 \mathrm{~mm}$ along different axes. In addition to computational errors, position errors consist of measurement, machining, and assembly errors. Measurement errors are caused by the calibration system, particularly position drift due to cylinder/valve leakage. In contrast with those of high-accuracy industrial robots, the machining and assembly of heavy-duty large-scale mechanical components exhibit relatively low accuracy. The operation of hydraulic manipulators in unstructured environments is human in the loop, and thus, the errors between the selected and measurement points shown in Fig. 14 are considered within the reasonable range.

\section{Conclusions}

In the current study, a 7-DOF redundant anthropomorphic hydraulic manipulator is developed for an intuitive kinematic operation in unstructured environments. This work is summarized as follows.

1) A hydraulic spherical RPY wrist structure designed as a revolute-universal chain by using a hybrid series-parallel mechanism is proposed. The 2-DOF pitch/yaw motion is achieved by two parallel cylinders with a 1RPRRR-1SPU mechanism. The roll motion is driven serially and separately by a hydraulic rotary cylinder. Compared with the RPR wrist, the proposed wrist can better resemble human wrist motion to achieve 
intuitive human-like operation. In addition, two rotary cylinders with high cost (vane type) or high friction (helical gear type) are replaced with linear servo cylinders to realize 2-DOF pitch/yaw motion.

2) On the basis of the spherical wrist, an anthropomorphic configuration of an HRM is designed, in which the third joint axis goes through the wrist center. An analytical inverse kinematic solution is derived via
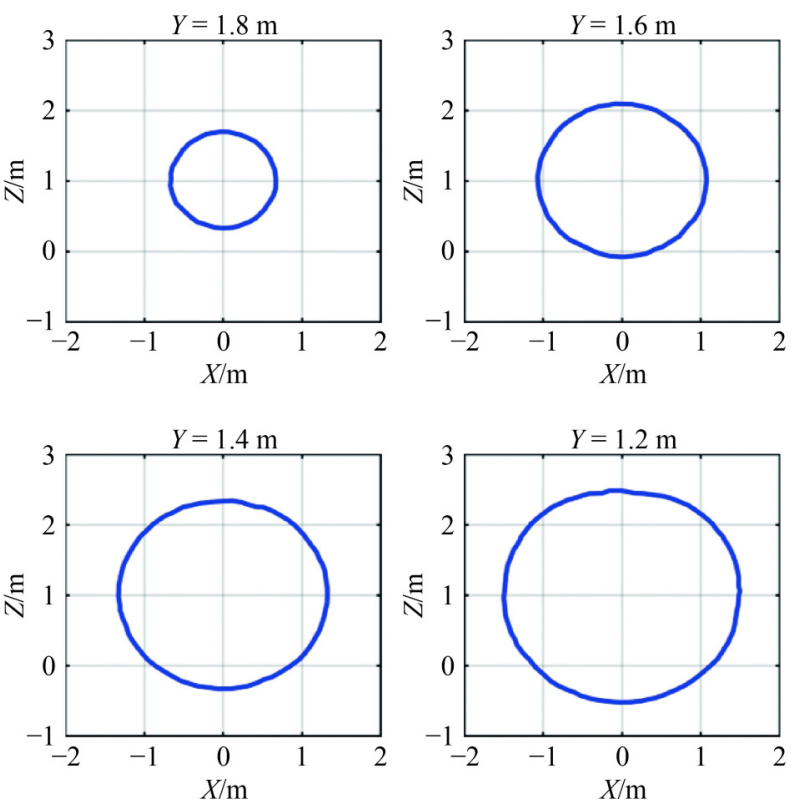

self-motion manifolds to describe redundancy uniquely. The forward and inverse kinematic computation methods are verified via numerical simulations and actual tests with a physical prototype.

The work is beneficial for designing hydraulic redundant manipulators with different motion workspaces or load-carrying capacities. Such manipulators can be used for rescue response, nuclear handling, or other applications in hazardous environments that require human real-time operations.
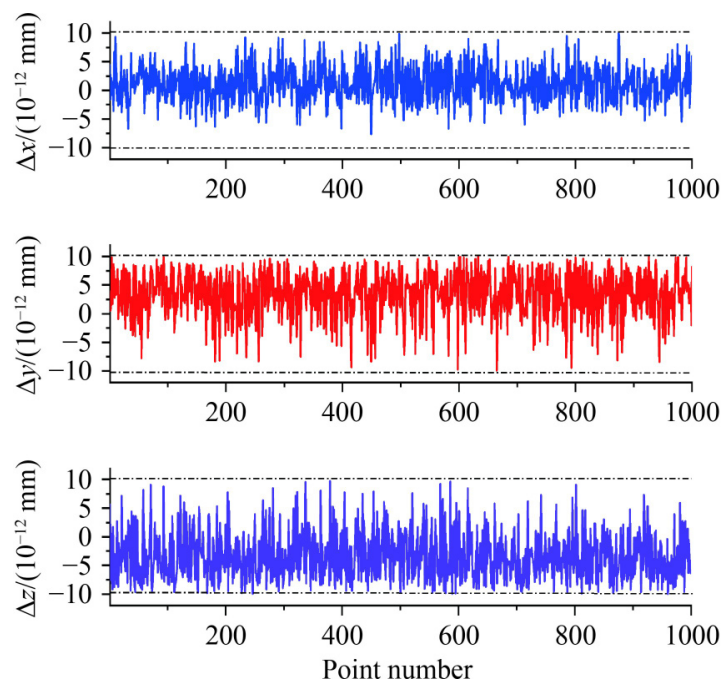

Fig. 12 Errors between the ideal and simulated trajectories.

Fig. 11 Ideal boundary trajectories on different $X O Z$ planes.
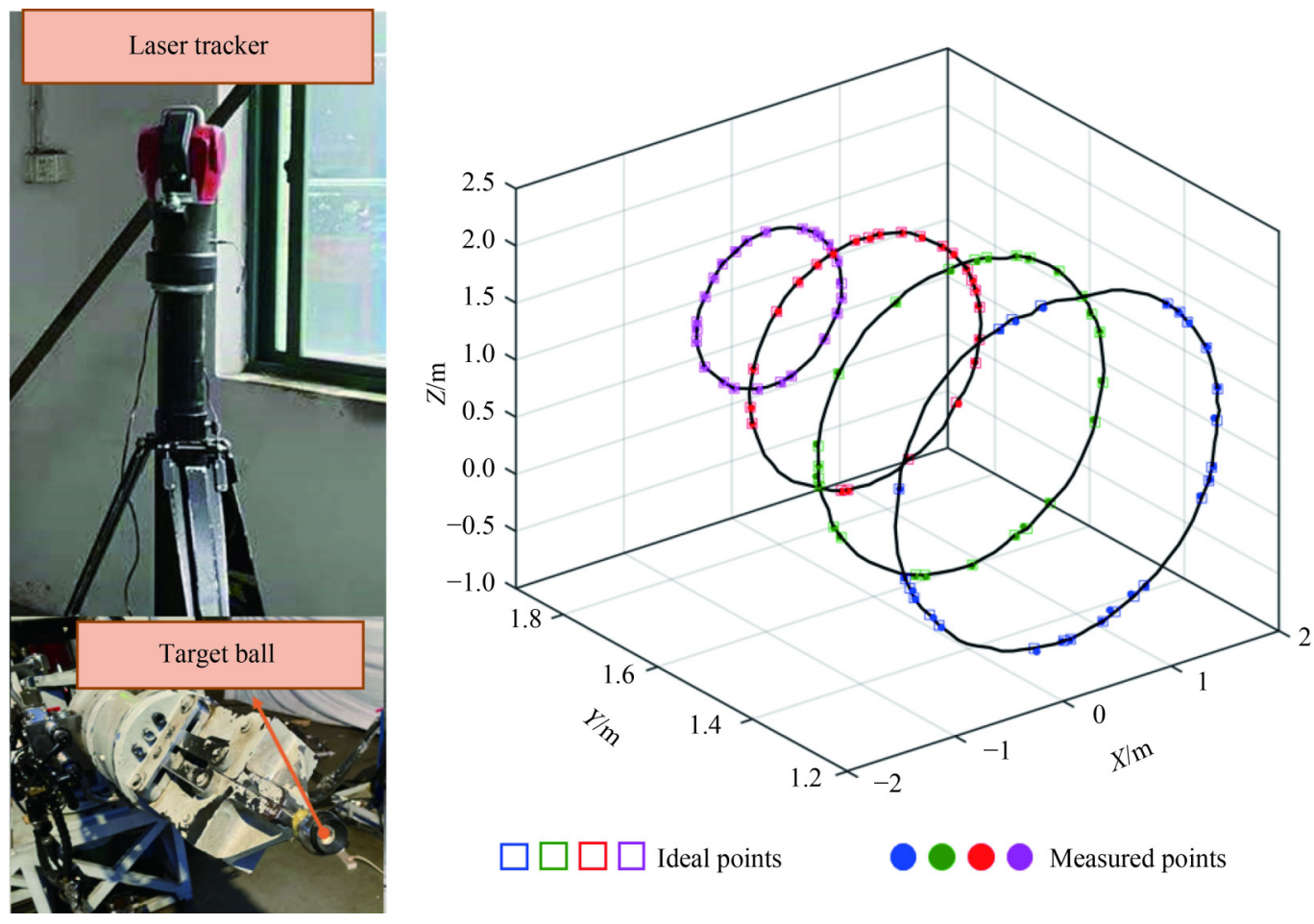

Fig. 13 Measurement system and ideal/measurement points. 

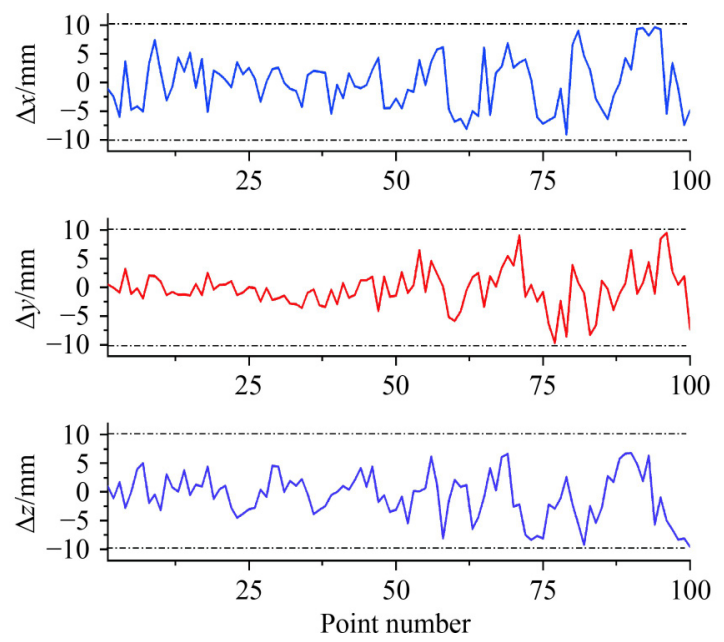

Fig. 14 Position errors between the selected and measurement points.

\section{Nomenclature}

Abbreviations

DH

DOF

HRM

RPR

RPY

SRS

Variables

$a_{i}(i=1,2, \ldots, 7)$
$b$
$d$
$d_{i}(i=1,2, \ldots, 7)$
$f$
$f_{j}(j=1,2, \ldots, g)$
$g$
$G$
$J_{x}$
$l_{0 \mathrm{p}}$
$l_{0 \mathrm{y}}$
$l_{\mathrm{e}}$

M

$n$

$O_{i}(i=1,2, \ldots, 7)$

$\boldsymbol{P}_{\mathrm{e}}$

$\boldsymbol{P}_{i}(i=A, B, \ldots, F)$

$q_{i}(i=1,2, \ldots, 7)$

Minimum yaw cylinder length

Distance from the wrist center to the endpoint of the manipulator

DOF of the equivalent mechanism

Number of links

Center point of the $i$ th joint

Position of the manipulator endpoint

Positions of Points $A-F$

Joint angle of the manipulator $\$_{i j}$

$\$_{i j}^{\mathrm{r}}$

${ }_{i}^{i-1} \boldsymbol{T}(i=1,2, \ldots, 7)$

$x_{A}, y_{A}, z_{A}$

$x_{C}, y_{C}, z_{C}$

$x_{\mathrm{e}}, y_{\mathrm{e}}, z_{\mathrm{e}}$

$x_{i}, y_{i}, z_{i}(i=1,2, \ldots, 7)$

$x_{\mathrm{p}}$

$x_{\mathrm{y}}$

$z_{4 l o w}, z_{4 \text { upp }}$

$\alpha_{i}(i=1,2, \ldots, 7)$

$\theta$

$\lambda$

$v$

$\varphi$

$\psi$
Motion screw of the $i$ th joint in the $j$ th limb

Reciprocal screw of the $i$ th joint in the $j$ th limb

Transformation matrices

Coordinate of Point $A$ along the $x$-, $y$-, and $z$-axis, respectively

Coordinate of Point $C$ along the $x$-, $y$-, and $z$-axis, respectively

Coordinate of the manipulator endpoint along the $x$-, $y$-, and $z$-axis, respectively

Coordinate of the center point of the $i$ th joint along the $x$-, $y$-, and $z$-axis, respectively

Pitch cylinder displacement

Yaw cylinder displacement

Lower and upper bounds of the $z$-coordinate of the elbow joint $R_{4}$, respectively

Link wrist angle between two adjacent joints

Pitch angle of the spherical wrist

Number of common constraint

Number of redundancy constraint

Roll angle of the spherical wrist

Yaw angle of the spherical wrist

Acknowledgements This work was supported in part by the National Natural Science Foundation of China (Grant Nos. 91748210 and 51922093), in part by the Natural Science Foundation of Chongqing, China (Grant No. cstc2020jcyj-msxmX0780), and in part by the Fundamental Research Funds for the Central Universities, China (Grant No. 2021CDJKYJH019).

Open Access This article is licensed under a Creative Commons Attribution 4.0 International License, which permits use, sharing, adaptation, distribution, and reproduction in any medium or format as long as appropriate credit is given to the original author(s) and source, a link to the Creative Commons license is provided, and the changes made are indicated.

The images or other third-party material in this article are included in the article's Creative Commons license, unless indicated otherwise in a credit line to the material. If material is not included in the article's Creative Commons license and your intended use is not permitted by statutory regulation or exceeds the permitted use, you will need to obtain permission directly from the copyright holder.

To view a copy of this license, visit http://creativecommons.org/licenses/ by $/ 4.0 /$.

\section{References}

1. Krotkov E, Hackett D, Jackel L, et al. The DARPA robotics challenge finals: results and perspectives. Journal of Field Robotics, 2017, 34(2): 229-240

2. Mattila J, Koivumaki J, Caldwell D G, et al. A survey on control of hydraulic robotic manipulators with projection to future trends. IEEE/ASME Transactions on Mechatronics, 2017, 22(2): 669-680

3. Sivčev S, Coleman J, Omerdić E, et al. Underwater manipulators: a review. Ocean Engineering, 2018, 163: 431-450 
4. Fang C, Ding X. A novel movement-based operation method for dual-arm rescue construction machinery. Robotica, 2016, 34(5): 1090-1112

5. Taylor C J, Robertson D. State-dependent control of a hydraulically actuated nuclear decommissioning robot. Control Engineering Practice, 2013, 21(12): 1716-1725

6. Klamt T, Kamedula M, Karaoguz H, et al. Flexible disaster response of tomorrow: final presentation and evaluation of the CENTAURO system. IEEE Robotics \& Automation Magazine, 2019, 26(4): 59-72

7. Kivelä T, Mattila J, Puura J. A generic method to optimize a redundant serial robotic manipulator's structure. Automation in Construction, 2017, 81: 172-179

8. Liang X, Wan Y, Zhang C, et al. Robust position control of hydraulic manipulators using time delay estimation and nonsingular fast terminal sliding mode. Proceedings of the Institution of Mechanical Engineers. Part I, Journal of Systems and Control Engineering, 2018, 232(1): 50-61

9. Li L, Xie L, Luo X, et al. Compliance control using hydraulic heavy-duty manipulator. IEEE Transactions on Industrial Informatics, 2019, 15(2): 1193-1201

10. Tadakuma R, Asahara Y, Kajimoto H, et al. Development of anthropomorphic multi-DOF mater-slave arm for mutual telexistence. IEEE Transactions on Visualization and Computer Graphics, 2005, 11(6): 626-636

11. Jacobsen S C, Smith F M, McCullough J. Teleoperated robotic system. US Patents, 019399A1, 2018

12. Ryu S M, Moon J W. Robot arm having hydraulic rotary actuators. US Patents, 0341228A1, 2017

13. Ding R, Cheng M, Jiang L, et al. Active fault-tolerant control for electro-hydraulic systems with an independent metering valve against valve faults. IEEE Transactions on Industrial Electronics, 2021, 68(8): 7221-7232

14. Hamiltona D T, Tesini A, Ranz R, et al. Progress in standardization for ITER remote handling control system. Fusion Engineering and Design, 2014, 89(9-10): 2409-2414

15. Zhang J, Li W, Yu J, et al. Development of a virtual platform for telepresence control of an underwater manipulator mounted on a submersible vehicle. IEEE Transactions on Industrial Electronics, 2017, 64(2): 1716-1727

16. Dominguez L M O. Optimization and redesign of a spherical wrist for a water hydraulic manipulator. Thesis for the Master's Degree. Tampere: Tampere University of Technology, 2011
17. Bajaj N M, Spiers A J, Dollar A M. State of the art in artificial wrists: a review of prosthetic and robotic wrist design. IEEE Transactions on Robotics, 2019, 35(1): 261-277

18. Shimizu M, Yoon W K, Kitagaki K, et al. Analytical inverse kinematic computation for 7-DOF redundant manipulators with joint limits and its application to redundancy resolution. IEEE Transactions on Robotics, 2008, 24(5): 1131-1142

19. Chu C Y, Xu J Y, Lan C C. Design and experiment of a compact wrist mechanism with high torque density. Mechanism and Machine Theory, 2014, 78: 65-80

20. Vischer P, Clavel R. Argos: a novel 3-DoF parallel wrist mechanism. International Journal of Robotics Research, 2000, 19(1): 5-11

21. Duan X, Yang Y, Cheng B. Modeling and analysis of a 2-DOF spherical parallel manipulator. Sensors (Basel), 2016, 16(9): 1485

22. Carricato M, Parenti-Castelli V. A novel fully decoupled twodegrees-of-freedom parallel wrist. International Journal of Robotics Research, 2004, 23(6): 661-667

23. Cammarata A. Optimized design of a large-workspace 2-DOF parallel robot for solar tracking systems. Mechanism and Machine Theory, 2015, 83: 175-186

24. Zhang C, Zhang L. Kinematics analysis and workspace investigation of a novel 2-DOF parallel manipulator applied in vehicle driving simulator. Robotics and Computer-Integrated Manufacturing, 2013, 29(4): 113-120

25. Bandara D S V, Gopura R A R C, Hemapala K T M U, et al. A multi-DoF anthropomorphic transradial prosthetic Arm. In: Proceedings of the 5th IEEE RAS/EMBS International Conference on Biomedical Robotics and Biomechatronics. São Paulo: IEEE, 2014, 1039-1044

26. Bridgwater L B, Ihrke C A, Diftler M A, et al. The Robonaut 2 hand-designed to do work with tools. In: Proceedings of 2012 IEEE International Conference on Robotics and Automation. Saint Paul: IEEE, 2012, 3425-3230

27. Huang Z, Zhao Y S, Zhao T S. Advanced Spatial Mechanism. Beijing: Higher Education Press, 2006 (in Chinese)

28. Tchoń K. Singularities of the Euler wrist. Mechanism and Machine Theory, 2000, 35(4): 505-515

29. Craig J J. Introduction to Robotics: Mechanics and Control. 3rd ed. Upper Saddle River: Prentice Hall, Pearson, 2005

30. Patel S, Sobh T. Manipulator performance measures-a comprehensive literature survey. Journal of Intelligent \& Robotic Systems, 2015, 77(3-4): 547-570 